\title{
Thinking on Indigenous Psychology of China Based on Western Psychology Culture
}

\author{
Qi Wang \\ Zhengzhou Vocational University of Information and Technology, Zhengzhou, 450046, China
}

Keywords: Psychology; Theoretical psychology; Empirical psychology; Theoretical evaluation system

\begin{abstract}
Currently, psychology of China is developing and being improved constantly. However, compared with western psychology, it still has many disadvantages and relies on western psychology to some extent but does not establish independent theoretical system. This thesis mainly starts with western psychology culture and its development process and compares it with development history of our psychology in order to help theories and practice of our psychology develop better.
\end{abstract}

\section{Introduction}

Psychology developed from the West in the beginning, so western psychology has important reference importance for other countries. Since 1950s, development of psychology has also covered many tortuous paths. During a long period, psychology depends on data analysis excessively, which reduces its scientificity instead. In the development history of psychology, some mature experience of western countries has extremely important reference significance for Chinese psychology. However, when we consult mature experience of western countries, we should have the ability to form our independent psychology system.

\section{Development history of western psychology}

Before modern psychology, philosophy had been included in scope of philosophy research. In the early days, study of philosophy on philosophy was not build based on practical analysis but just carried out related researches on people's mentality by theoretical study. In modern times, some distinguished psychologists represented by Hobbes and Locke appeared. Their analyses of human mentality are implemented are multi-angle and multi-layered. However, since they did not combine with demonstration to analyze human psychology, people just call them philosophers.

It is after the 1850s that Wundt a German psychologist did psychic-reflex experiments on a batch of students, which started the first case that experimental science was used to study psychology. Since then, psychology had not been a part of philosophy but an independent subject. Because research methods were more rigorous, development road of psychology became more scientific and more logical. Psychology during this period was affected by natural science and relied on life experience in the aspect of theoretical formation. As it depended on experience too much, theories that were not established based on observation or not verified by practical life were denied. During this period, development of psychology was limited to some extent since concluding methods of psychology were not mature enough.

In this period, many psychologists attached excessive importance to demonstration and even completely ignored importance of theory. Some psychologists even thought that theories were redundant in psychological study. Since psychologists worshipped experience excessively but ignored theoretical study, study of psychology on development and changes in people's mentality was not profound enough during this period although it made a contribution.

In the 20th century, microphysics developed to a peak, which drove psychology to develop from empirical experience to thought experiments. During this period, psychologists proposed this opinion: system of psychology should include two parts, i.e., theory and demonstration and the two parts supplement and promote each other. Appearance of the opinion made theory and demonstration realize logical unification, which not only laid a solid foundation for theoretical 
development of psychology but also opened research methods of psychology.

\section{Development process of Chinese psychology}

Chinese psychology starts late and develops slow, so it is much more backward than western psychology to some extent. However, according to development history of Chinese psychology, we can still find that indigenous psychology of China has obtained many achievements. The development history of Chinese psychology mainly includes three stages:

\subsection{Stage 1: sprouting}

Sprouting history of Chinese psychology was started at the beginning of new China. At that time, a large number of splendid psychologists appeared, such as Chen Daqi, Gao Juefu and Guo Renyuan. For historical reasons, these psychologists suffered strikes while doing scientific researches. Nevertheless, they did not fear difficulty but obtained many precious achievements.

\subsection{Stage 2: stagnation}

The reason why Chinese psychology stagnated was related to death of the older generation of psychologists. Since China started Reform and Opening-up just now in the later period of the last century, the younger generation began to be restless and uneasy so that they could not get down to study knowledge. Thus, psychological talent stayed in a stage of temporary shortage and talent fault even appeared.

2.3 Stage 3: all flowers bloom together

Since the 21 st century, a number of outstanding psychological researches have devoted themselves to exploring psychology with leadership of newly appearing psychologists, for instance, Ya Haosheng. Chinese psychological study has walked out its valley finally and showed a prosperous scene since then. As researches deepen, psychological system is becoming mature and mature. Up to now, Chinese experts have obtained a great many of achievements in psychology.

Although Chinese psychology has formed theoretical system preliminarily, there is still a gap between it and western psychology, which is reflected by both research scale and methods and participants of researches.

\section{The reason why there is a gap between Chinese psychology and western psychology}

\subsection{Lacking establishment of subject system}

Scientization level of the research can be improved, psychological study can be developed to be stronger and outstanding talent can be attracted to do specialized researches only when disciplinization is carried out for psychology. However, the current situation is that research effort of China is not powerful enough or resources that can be used are not rich sufficiently. Accordingly, some psychological influence is relatively small. About importance of subject system for psychology, some scholars said, 'Being similar to the situation that basic science should be equipped with laboratories, each college should arrange some scientific research centers in its Psychology Department. In doing so, more researchers can be cultivated and development and research group of psychology can be expanded as well'.

At present, many universities and research centers in China do not realize importance of subject system. Thus, they do not establish specialized positions for psychology. Besides, when Psychology Department of each university supplements its teachers, it rarely chooses doctors of the subject. At the same time, when it recruits Psychology postgraduate students, it mainly focuses on students' researches on experimental psychology, which causes imbalance of psychological researchers, i.e., they attach importance to demonstration but neglect theories.

In the foregoing content, we have mentioned that psychology must walk on two legs, i.e., demonstration and theory should coexist. Otherwise, it is impossible to obtain sustainable development or get food effect in practical application. Western scholars have realized necessity of walking on two legs, Chinese indigenous psychologists should learn from western experts in this aspect.

3.2 Lacking independent theoretical system 
Currently, researches of Chinese psychology are established based on existing research achievements of the west. All research methods, research scopes and judgment criteria rely on the series of achievements that western psychology has formed. Such a practice may make full use of existing resources, reduce tortuous paths in development process and thus save cost and time. Nevertheless, disadvantages are obvious.

In western value, people's ideology can be divided into three parts, i.e., knowledge, emotion and consciousness. Western people think the three can be separated and independent of one another. They also hold such an opinion when they carry out psychological researches. Some experts deem that such a kind of cognition materialize people, while people's psychological process is a quite complicated process and is affected by joint effect of several factors, which is contradictory with western value.

Pan Shu a famous psychologist deems that people are not cold but have life, consciousness and emotion in traditional theories of Chinese psychology. People's mental mechanism is not phenomenon that is accurately rational but is a result of joint effect of people's knowledge, emotion and consciousness. Thus, when psychological researches are carried out, it is essential to include the three elements in the research scope. The conclusion drawn in this way is the most reliable and accords with practical situations.

However, since Chinese psychology is affected by the west excessively, modern psychology of China does not pay attention to interaction among people but puts research emphasis on interaction between people and objects.

It is nothing else but means western psychology leads Chinese psychology by the nose. Indigenous psychology of China cannot make considerable progress or independent system cannot be generated if the negative impact is not eliminated.

\section{Establishing psychological system of China}

In accordance with current situations, there are some shortages in psychological research of China. To eliminate these shortages, it will be essential to spend lots of manpower and financial resources in a long term. In order to get development, Chinese psychology cannot absorb nutrition from western psychology. In the aspect of demonstration, western psychology has obtained quite abundant experience, and the effect achieved by its scientific experiments is amazing. In the aspect of theoretical study, western countries have many methods to do theoretical analysis, including analysis, conclusion, hypothesis, comprehension, analogy, deduction, abstraction and inference etc. These methods explore and study various mental phenomena and all kind of professional systems of psychology are formed finally.

In accordance with the two aspects, Chinese psychology and western psychology connect with each other. However, by combining with different cultures and national conditions, we cannot copy theories or achievements of western psychology overall but should establish a system that is related to western psychology but independent of western psychology simultaneously.

\subsection{Two driving patterns}

Throughout development history of world science, we can find that there are two approaches in the development process of all subjects. One approach is 'concept driving'. It means we should follow the order from top to bottom when we summarize theories. When inference is carried out, conclusions can be drawn by starting with pure thinking without experience via various kinds of inference or analytical methods. Secondly, the other approach is 'data driving'. It means the order from bottom to top should be followed when empirical data are collected. Conclusions drawn by this method are more rational and accord with mathematical logic to a larger extent.

The two driving patterns are appropriate for both western psychology and study and development of Chinese psychology.

4.2 Ways to establish and complete system of Chinese psychology

When indigenous psychology carries out studies according to the two approaches, there are three situations that attention must be paid to. Firstly, choose achievements and research methods of foreign psychology conditionally but do not copy them completely. Secondly, inject more unique 
achievements of China based on western psychology and explore our own research methods. Thirdly, do examine the three relations comprehensively, i.e., the relation between theory and demonstration, the relation between learning and innovation as well as the relation between introspection and construction.

Firstly, select western psychology and learn the content that is helpful for development of the system of Chinese indigenous psychology. Since the middle and later period of the 20th century, theoretical psychology has still use philosophical methods for research chronically but has been different from previous speculation. Additionally, western psychology is reducing subjectivity in psychological study gradually and paying more attention to objective researches according to current situations. Take 'meta-analysis' for example. The method concludes laws based on conclusions of objective data. The research method is more rigorous and standard and also provides models and enlightenment for other experts' researches.

Secondly, it is essential to insist on covering the road of originality. The older generation of psychologists has put forward many useful models and theories, but most of these theories are independent and have no much significance for reference. Nowadays, indigenous psychology of China needs the 'meta-theory' that has guiding effect on psychological study. Achievements of the older generation of psychologists are not enough to undertake the heavy responsibility of the 'meta-theory'. Thus, researches of China should be placed on construction of the 'meta-theory' at present. The reason for this is that the 'meta-theory' can give distinct directions to development of psychology, which makes scholars find guiding light in complicated academic environment.

In the aspect where originality is enhanced, we should achieve the following aspects. Firstly, theoretical research should be people-oriented. Secondly, the will to do theoretical researches cannot be shaken. Thirdly, theories should be combines with demonstration. Fourthly, theoretical researches should have both criticalness and construction. Fifthly, researchers should implement close cooperation. Sixthly, theoretical methods may be diversified.

Thirdly, when indigenous psychological study is carried out, it is essential to examine the three relations comprehensively, i.e., the relation between theory and demonstration, the relation between learning and innovation as well as the relation between introspection and construction. Practice and theory are inseparable. Practice is a basis of theories, and theories also guide successful implementation of practice. Study and innovation supplement each other. When Chinese psychology is undeveloped, learning is inevitable. However, changeless study lost significance of study itself, so we must carry out innovation for related knowledge based on learning, which can make Chinese psychology comparable with western psychology. The situation that construction ought to be performed when introspection is implemented aims at prevent psychological from suffering standstill.

\section{Conclusion}

Development road of western psychology tells us that development road of any subject is not accomplished in one action. To make indigenous psychology of China obtain development and perfection, we should not only learn from western psychology but also insist on covering the road of originality and make wisdom of Chinese researches be developed sufficiently.

\section{References}

[1] Ma Tianyu and Meng Weijie. Indigenous Psychology: Understanding Different Perspectives of 'Mentality' - Surpassing of Indigenous Psychology for Western Mainstream Psychology [J]. Theoretical Discussion: 2006,03:57-59.

[2] Song Xiaodong and Ye Haosheng. Indigenous Psychology and Multi- culturalism — Meeting in Theoretical Prospect of Human Psychology [J]. Journal of Xuzhou Normal University (Philosophy and Social Science Edition), 2008,01:112-116.

[4] Ge Lujia. Theoretical Construction of New Mind-Nature Psychology — One New-century Choice of Theoretical Innovation of China's Indigenous Psychology, Jilin University Journal Social 
Sciences Edition, 2005,05:142-151.

[5] Chen Yingmin and Zhou Pizhen. Between Globalization and Localization: Construct A Multicultural Opinion on Modern Psychology [J], Journal of Shandong Teachers' University (Humanities and Social Sciences), 2005,03:132-135.

[7] Zhang Haiyu. Exploration on Difficulty and Outlet of China's Indigenous Psychology [J]. Journal of Qinghai Nationalities University, 2010,03:98-101.

[8] Ge Lujia. Scientization and Localization of Chinese Psychology - Cross-century Theme of China's Psychology Development [J]. Jilin University Journal Social Sciences Edition, 2002,02:5-15. 